\title{
Early Morbidity and Mortality Following In Utero Exposure to Selective Serotonin Reuptake Inhibitors
}

\section{A Population-Based Study in Western Australia}

\author{
Lyn Colvin, ${ }^{1,2}$ Linda Slack-Smith, ${ }^{2}$ Fiona J. Stanley ${ }^{1}$ and Carol Bower ${ }^{1,3}$
}

1 Telethon Institute for Child Health Research, Centre for Child Health Research, The University of Western Australia, Perth, WA, Australia

2 School of Dentistry, The University of Western Australia, Perth, WA, Australia

3 Western Australian Register of Developmental Anomalies, Perth, WA, Australia

\section{Abstract}

Background: The early years of life have a profound effect on a child's developmental pathway. The children born to mothers suffering from depression may be at risk of increased morbidity and mortality in the first years of life.

Objective: The objective of this study was to investigate the hospital admissions and mortality of children whose mothers were dispensed a selective serotonin reuptake inhibitor (SSRI) during their pregnancy.

Methods: This was a population-based study of all pregnancy events in Western Australia (WA) from 2002 to 2005. The study used linkable state health administrative data from the WA Data Linkage System (WADLS) and the national Pharmaceutical Benefits Scheme (PBS), enabling birth outcomes, hospital admissions and deaths to be ascertained for the children of women dispensed an SSRI during their pregnancy.

Results: There were 3764 children born to 3703 women who had been dispensed an SSRI during their pregnancy (3.8\% of all pregnancies in WA, 2002-5), and 94561 children born to 92995 women who had not been dispensed an SSRI. Mean birth weight, length and APGAR score at 5 minutes were significantly lower in children of women dispensed an SSRI, regardless of whether the SSRI was dispensed in trimester 1, or, trimester 2 or 3 only. $0.9 \%$ of the live born children in the SSRI group had died before the age of 1 year compared with $0.5 \%$ of the non-SSRI group (odds ratio [OR] 1.8; $95 \%$ CI 1.3, 2.6). Before the age of 2 years, $42.9 \%$ of the children in the SSRI group had been admitted to hospital after their birth admission, compared with $34.1 \%$ of the non-SSRI group (OR 1.4; 95\% CI 1.3, 1.6). The most common reason for admission to hospital was acute bronchiolitis (OR 1.6; 95\% CI 1.3, 1.8), with an increased risk seen in children of mothers who did not smoke during their pregnancy (OR 1.7; 95\% CI 1.4, 2.0). 
Conclusions: The children in the SSRI group were more likely to be admitted to hospital in the first years of life, and this may reflect their prenatal exposure to SSRIs, be related to maternal depression, or SSRI use may be a proxy for an environmental exposure such as smoking, or a combination of these factors. Although the numbers of deaths in the first year of life were small, the increased risk of death in the first year of life in the SSRI group (OR 1.8; 95\% CI 1.3, 2.6) is a new finding and should be investigated further.

\section{Background}

The highest rates for depression occur in women between the ages of 25 and 44 years, ${ }^{[1]}$ and these years account for the majority of the child-bearing period. The largest, and most recent, survey of pregnant women in Australia found approximately $9 \%$ of the women surveyed fulfilled the diagnostic criteria for depression. ${ }^{[2]}$ A study of women with a prior history of major depression found that those who discontinued antidepressant treatment were five times more likely to relapse during pregnancy than those who continued treatment during pregnancy. ${ }^{[3]}$ The part played by the underlying depression in the mother in relation to neonatal outcomes is not clear. Studies by Field et al. ${ }^{[4,5]}$ considered relationships between prenatal depression and decreased foetal growth and between cortisol and shorter gestation but did not distinguish between mothers taking antidepressants and those that did not.

Selective serotonin reuptake inhibitors (SSRIs) are used in the treatment of depression and in anxiety disorders such as panic disorder, as well as chronic pain. Studies have shown that SSRIs readily cross the placenta, ${ }^{[6]}$ are present in amniotic fluid ${ }^{[7]}$ and newborns exposed to them in utero may experience withdrawal behaviours after birth. ${ }^{[8,9]}$ A review by Belik ${ }^{[10]}$ found the available information related to the clinical symptoms of infants exposed to SSRIs in utero is limited to small cohort studies, case reports and to international collaborative programmes that monitor adverse reactions to drugs, such as the USA FDA and the WHO Collaborating Centre for International Drug Monitoring. There have been some follow-up studies of the children exposed to SSRIs in utero. A review of neurodevelopmental outcomes by Gentile and Galbally ${ }^{[1]}$ found there were very few longitudinal studies of children exposed in utero to antidepressants. Another review of late pregnancy exposure to antidepressants found a constellation of recurrent symptoms in the first days of life in the newborns and suggested that the term 'prenatal antidepressantexposure syndrome' be used to describe it. ${ }^{[12]}$

Early hospital admissions, particularly after the birth admission, in children exposed to SSRIs in utero have not been investigated. We have previously reported the dispensing patterns, pregnancy and birth outcomes of women dispensed an SSRI during their pregnancy in a populationbased study relating to all pregnancy events in Western Australia (WA) from 2002 to 2005 (N= 96968 pregnancies resulting in a birth). ${ }^{[13]}$ There were 3703 women (3764 children) dispensed an SSRI during their pregnancy. $42.3 \%$ of these women were dispensed an SSRI in each trimester, $75.4 \%$ of the women were dispensed an SSRI in consecutive trimesters and $97.6 \%$ of the women used the same SSRI throughout the first trimester without switching. However, we did not report the effects of SSRI by trimester of exposure, nor outcomes for the child beyond birth.

\section{Objectives}

Using data linkage of population-based data, we investigated the early hospital admissions and mortality for the children of the women described in our previous paper ${ }^{[13]}$ and compared hospitalisation and mortality rates of these children with rates for all other children whose mothers were not dispensed an SSRI. 


\section{Methods}

\section{Study Design}

This was a population-based study relating to all pregnancy events in WA from 2002 to 2005. The study used linkable state health administrative data from the WA Data Linkage System (WADLS, which contains data from the Hospital Morbidity Data System, the Midwives' Notification System, the WA Registry of Births and Deaths and the WA Register of Developmental Anomalies) and the national Pharmaceutical Benefits Scheme (PBS), enabling birth outcomes, hospital admissions and deaths to be ascertained for the children of those women dispensed an SSRI during their pregnancy. The PBS is a claims database that includes $80 \%$ of all prescriptions dispensed in Australia. The linkages and methodology have been described previously. ${ }^{[13-15]}$

\section{Setting}

During 2002-5 there were six generic medicines listed on the PBS that fell under the category of SSRI (citalopram hydrobromide, escitalopram oxalate, fluoxetine hydrochloride, fluvoxamine maleate, paroxetine hydrochloride and sertraline hydrochloride). SSRIs are only available in Australia on the PBS as a 'Restricted Benefit' for major depressive disorders, obsessive-compulsive disorder (fluoxetine, fluvoxamine, paroxetine, sertraline) or panic disorder (paroxetine, sertraline) ${ }^{[16]}$ and are not available over the counter.

The International Statistical Classification of Diseases and Related Health Problems, Tenth Revision, Australian Modification (ICD-10-AM)/ Australian Classification of Health Interventions $(\mathrm{ACHI})$ is the system used to code inpatient episodes of care in all Australian public and private hospitals. The ICD-10-AM is used to code diseases and problems. ${ }^{[17]}$

\section{Participants}

As hospital admission data were available from 2002 to 2006, admissions were analysed for all children to the age of 1 year, and to 2 years for those children born 2002-4. Characteristics of the mothers and children are from the midwives' records.

\section{Major Variables and Data Sources Used in Outcome Measurements}

The hospital admission data include a principal diagnosis and up to 20 additional diagnoses as recorded on the discharge records. The codes are based upon ICD-10-AM.$^{[17]}$ According to the Hospital Morbidity Data System coding guide that was current during the study, ${ }^{[18]}$ additional diagnoses with the following characteristics need to be coded:

- require therapeutic treatment;

- require performance of a diagnostic procedure;

- increase nursing care and/or monitoring; or,

- may extend the length of stay in hospital.

The admission data were validated and records were merged if the child was re-admitted within a day for the same principal diagnosis, to the third ICD-10-AM digit. This will also reflect a more clinical view of admissions as hospital transfers will be recognized as the same episode of care and counted as one admission. Lengths of stay (LOS) were calculated as the number of days from admission to discharge for the same episode of care. Admissions discharged on the same day were allocated a LOS of 0.5 day.

The Midwives' Notification System was used to determine stillbirths. The WA Registry of Deaths provided the date of death for all deaths.

The Australian Bureau of Statistics has released Socio-Economic Indexes for Areas (SEIFA) based on the information collected in the 5-yearly Census of Population and Housing. These indexes are widely used measures of relative socio-economic status at a small geographical area level. The indexes rank and identify areas that are relatively more, or less, disadvantaged. They provide contextual information about the area in which a person lives. The indexes have been obtained by a principal components analysis that summarizes the information from a variety of social and economic variables, calculating weights that will give the best summary for the underlying variables. The categories of variables include income, education, employment, occupation and housing. The mean rank is $1000 .^{[19]}$ The SEIFA values 
in this study are based upon the 2001 Australian Census of Population and Housing.

The variable, Proportion of Optimal Birth Weight (POBW), was calculated as a measure of the appropriateness of foetal growth. ${ }^{[20]}$ POBW is independent of gestational age and takes into account the major non-pathological determinants of intrauterine growth. POBW is the ratio of the observed to the 'optimal' birth weight; the latter estimated from a regression equation including terms for gestational duration, maternal height, parity and infant sex, derived from a total population of singleton births that excluded those exposed to risk factors for intrauterine growth restriction, including maternal smoking. ${ }^{[21]}$

\section{Statistical Methods}

Comparisons were made between the children of women dispensed an SSRI at any time during pregnancy and those not dispensed an SSRI at any time during their pregnancy. ORs with $95 \%$ confidence intervals were calculated to assess the strength of association of the child characteristics, admission rates and LOS. Stepwise logistic regression using the $\mathrm{SAS}^{\circledR}$ (SAS Institute Inc., Cary, NC, USA) procedure, PROC LOGISTIC, was used to adjust ORs. T-tests were used to compare means where appropriate. Some comparisons were made using just the term births to avoid the potential effects of preterm birth. The infant mortality rate (deaths to age 1 year in live births) in Australia for the period 2000-5 was 4.959 per 1000 live births. ${ }^{[22]}$ There were 3738 live births in the SSRI group. Our study had $88 \%$ power to detect 9 per 1000 live births. ${ }^{[23]}$

The WADLS uses the Automatch ${ }^{\circledR}$ software package (Matchware Technologies Inc., Kennebunk, ME, USA) with probabilistic matching based upon medical record number, surname, first given name and initial, date of birth, sex and address as the principal matching fields. Missed links have been estimated at $0.11 \%$. $^{[2]}$ The WADLS has been validated previously ${ }^{[24,25]}$ and has been used extensively for health research. ${ }^{[26]}$ All records for this study were also validated internally. For example, sex and dates of birth or death were checked across each source. The datasets were analysed using SAS $^{\circledR} /$ STAT $^{\circledR}$ software, version 9.2 of the SAS System for Windows (SAS Institute Inc., Cary, NC, USA). The researchers received all data in a de-identified form from the WADLS.

\section{Ethics Approvals}

This project has approval from the Human Research Ethics Committees of The University of WA and the WA Department of Health.

\section{Results}

From 2002 to 2005 in WA there were 96698 pregnancies resulting in the birth of 98325 infants. There were 16294 dispenses of an SSRI.

Characteristics of the Children and Their Birth Outcomes, by Trimester of Exposure

There were 3764 children born to 3703 (3.8\% of all pregnancies in WA, 2002-5) women who had been dispensed an SSRI during their pregnancy, and 94561 children born to 92995 women who had not been dispensed an SSRI during pregnancy. The characteristics of the women and children are reported in table I showing the differences between women dispensed an SSRI in trimester 1 (T1, N=2654), or those dispensed an SSRI in trimester 2 or 3 only (T2 or T3, N= 1049), and all other women. The mean number of dispenses per pregnancy was 2.2 in T1 and 4.4 for any time in pregnancy. An APGAR score at 5 minutes of less than 6 was more likely in children of women dispensed an SSRI in T1 (OR 1.8; $95 \%$ CI $1.4,2.3)$ than those dispensed only in T2 or T3 (OR 1.2; 95\% CI 0.8, 2.0) when compared with the children in the non-SSRI group. Preterm birth was also more likely in the T1 group (adjusted OR 1.6; 95\% CI 1.3, 1.9) than those in T2 or T3 only (adjusted OR 1.3; 95\% CI 1.0, 1.7), after adjusting for a previous preterm birth, smoking, SEIFA, parity and maternal age, and considering only singletons. When the risk of a birth weight $<2500 \mathrm{~g}$ was adjusted for gestational age, whether the mother smoked during her pregnancy, SEIFA, sex, parity and maternal height, dispenses in T2 or T3 only remained significant (adjusted OR 1.5; $95 \%$ CI $1.1,2.0)$. 


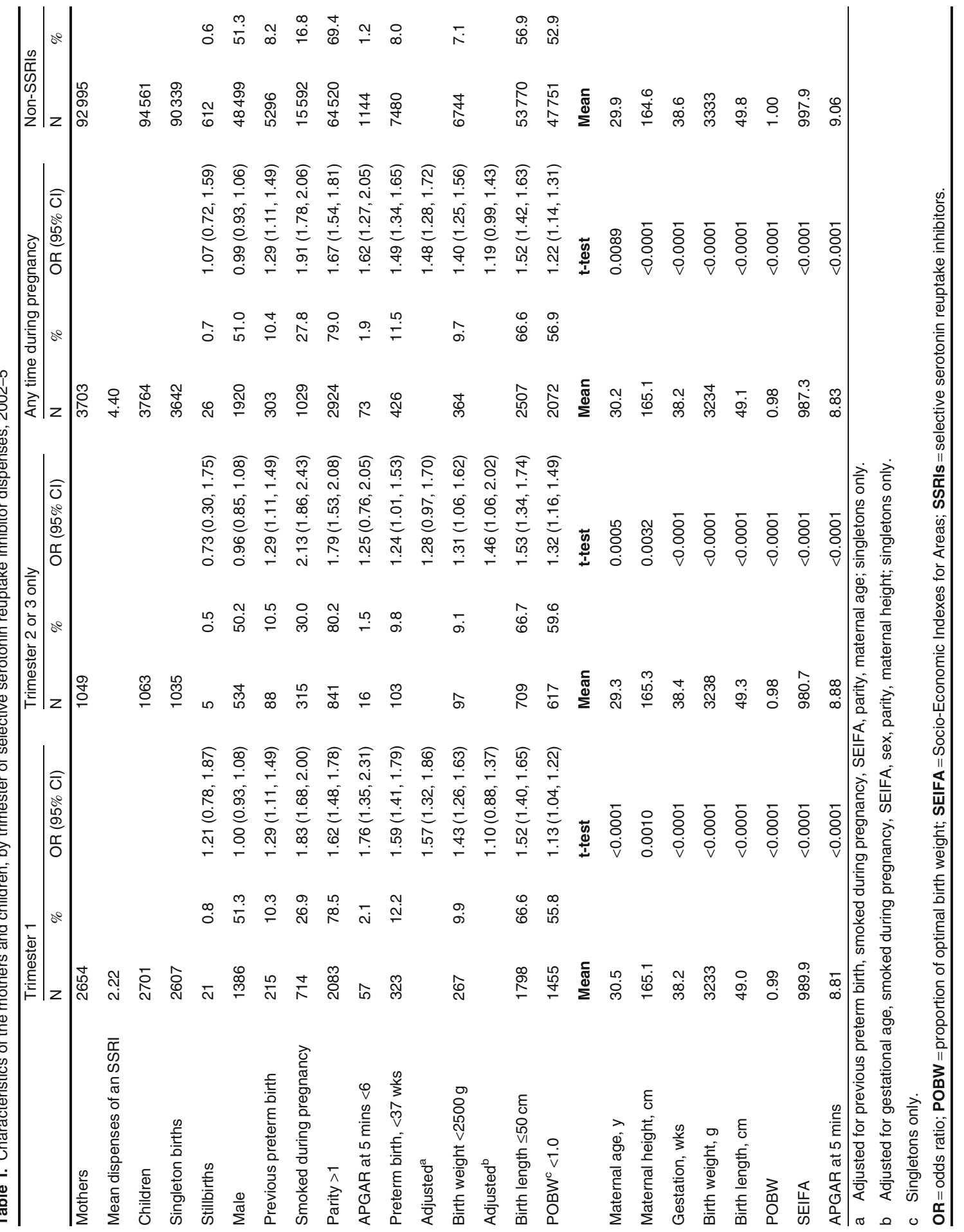


1.1; 95\% CI 0.7, 1.6). There were no differences between the children with a major birth defect, male children or the preterm births to adequately describe this higher mortality risk. The codes used to describe the deaths were reviewed. 'P01 fetus and newborn affected by maternal complications of pregnancy' and 'P07 disorders related to short gestation and low birth weight, not elsewhere classified' were the most common codes used in the deaths of the children in the SSRI group.

Death rates in the first year of life in children live born were greater for each individual SSRI as well as overall (table II). Although the number of deaths is very small, a statistically significant increased risk was seen in the children of mothers dispensed paroxetine (OR 2.2; 95\% CI 1.0, 4.6), escitalopram (OR 3.5; 95\% CI 1.3, 9.5) and fluvoxamine (OR 4.5; 95\% CI 1.4, 14.2). Two of the three children in the fluvoxamine group had mothers also dispensed escitalopram $(\mathrm{N}=1)$ and citalopram $(\mathrm{N}=1)$.

There were no deaths in the SSRI group during the second year of life.

\section{Admissions in the First 2 Years of Life}

A summary of the admissions for birth, intensive care units (ICU) and non-birth admissions is presented in table III. The mean LOS for the birth admission was significantly longer in the children in the SSRI group (5.2 vs 4.9 days; t-test: 0.0071 ); there were no other significantly different LOS between the two groups. The children in the SSRI group were also at a greater risk of a nonbirth admission or an ICU admission (ranging from $31 \%$ to $48 \%$ increased risk) during the first 2 years of life.

\section{Birth Admissions}

Excluding the regular admission coded for a birth ('Z38 live born infants according to place of birth'), the ten most common diagnoses recorded on the birth admissions for term births of children in the SSRI group are presented in table IV. The most common diagnosis recorded was ' $\mathrm{Z} 03$ medical observation and evaluation for suspected diseases and conditions' (OR 1.6; 95\% CI 1.4, 1.7). The next most common diagnosis recorded

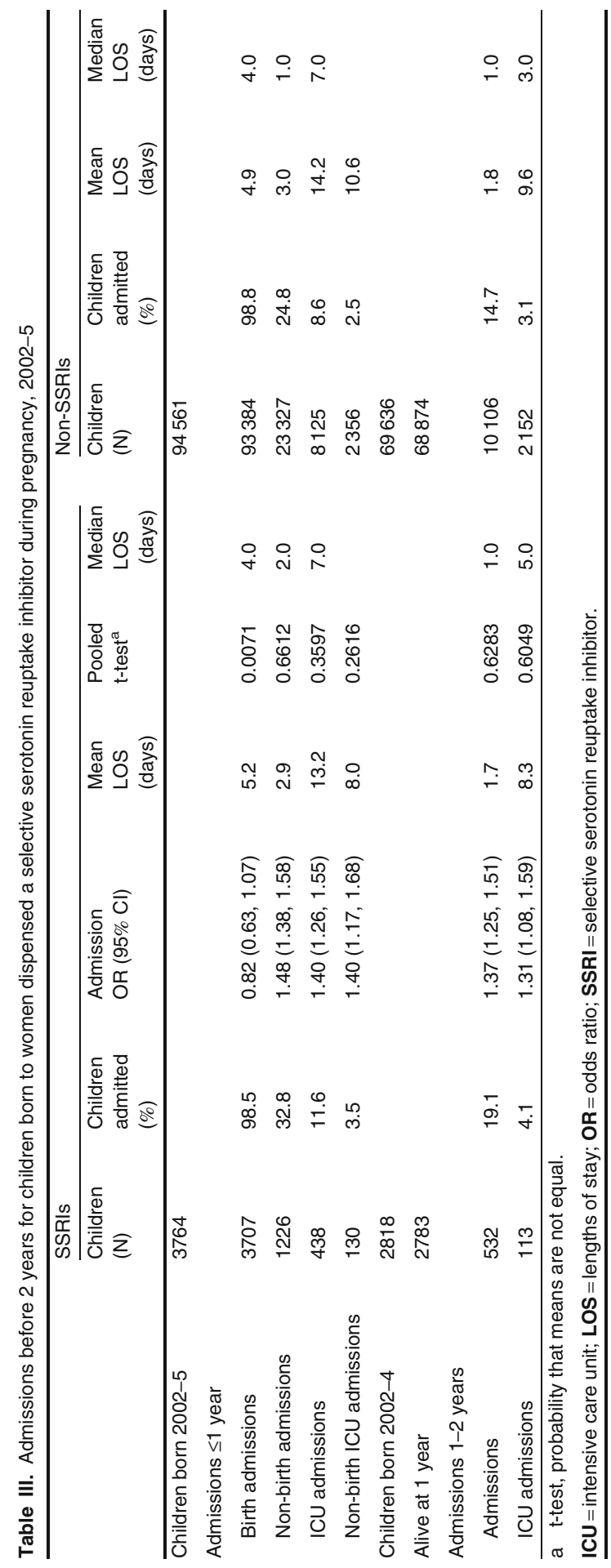


Table IV. Leading ten diagnosis codes of term birth admissions for children in the selective serotonin reuptake inhibitor group, by number of children admitted

\begin{tabular}{|c|c|c|c|c|c|}
\hline \multirow[t]{2}{*}{ Diagnosis codes } & \multicolumn{2}{|c|}{$\begin{array}{l}\begin{array}{l}\text { SSRIs } \\
\mathrm{N}=3297\end{array} \\
\end{array}$} & \multicolumn{3}{|c|}{$\begin{array}{l}\text { Non-SSRIs } \\
\mathrm{N}=86110 \\
\end{array}$} \\
\hline & $\mathrm{N}$ & $\%$ & $\mathrm{~N}$ & $\%$ & OR $(95 \% \mathrm{Cl})$ \\
\hline Z03 Medical observation and evaluation for suspected diseases and conditions & 430 & 13.0 & 7540 & 8.8 & $1.56(1.41,1.73)$ \\
\hline P22 Respiratory distress of newborn & 255 & 7.7 & 3986 & 4.6 & $1.73(1.51,1.97)$ \\
\hline Adjusted for Caesarean delivery & & & & & $1.71(1.50,1.96)$ \\
\hline P92 Feeding problems of newborn & 217 & 6.6 & 4881 & 5.7 & $1.17(1.02,1.35)$ \\
\hline Z29 Need for other prophylactic measures & 162 & 4.9 & 2985 & 3.5 & $1.44(1.22,1.69)$ \\
\hline P96 Other conditions originating in the perinatal period & 157 & 4.8 & 1086 & 1.3 & $3.91(3.30,4.65)$ \\
\hline P39 Other infections specific to the perinatal period & 151 & 4.6 & 3668 & 4.3 & $1.08(0.91,1.27)$ \\
\hline P28 Other respiratory conditions originating in the perinatal period & 147 & 4.5 & 2099 & 2.4 & $1.87(1.57,2.22)$ \\
\hline P59 Neonatal jaundice from other and unspecified causes & 139 & 4.2 & 4604 & 5.3 & $0.78(0.66,0.93)$ \\
\hline P05 Slow fetal growth and fetal malnutrition & 78 & 2.4 & 1356 & 1.6 & $1.51(1.20,1.91)$ \\
\hline P70 Transitory disorders of carbohydrate metabolism specific to fetus and newborn & 78 & 2.4 & 1322 & 1.5 & $1.55(1.23,1.96)$ \\
\hline P20-P29 Respiratory and cardiovascular disorders specific to the perinatal period & 455 & 13.8 & 7049 & 8.2 & $1.80(1.62,1.99)$ \\
\hline Adjusted for Caesarean delivery & & & & & $1.79(1.62,1.99)$ \\
\hline P20 Intrauterine hypoxia & 17 & 0.5 & 294 & 0.3 & $1.51(0.93,2.47)$ \\
\hline P21 Birth asphyxia & 32 & 1.0 & 382 & 0.4 & $2.20(1.53,3.16)$ \\
\hline P22 Respiratory distress of newborn & 255 & 7.7 & 3986 & 4.6 & $1.73(1.51,1.97)$ \\
\hline P24 Neonatal aspiration syndromes & 15 & 0.5 & 228 & 0.3 & $1.72(1.02,2.91)$ \\
\hline P25 Air leak syndrome originating in the perinatal period & 6 & 0.2 & 134 & 0.2 & $1.17(0.52,2.65)$ \\
\hline P28 Other respiratory conditions originating in the perinatal period & 147 & 4.5 & 2099 & 2.4 & $1.87(1.57,2.22)$ \\
\hline P29 Cardiovascular disorders originating in the perinatal period & 47 & 1.4 & 652 & 0.8 & $1.90(1.41,2.55)$ \\
\hline Adjusted for Caesarean delivery & & & & & $1.89(1.40,2.54)$ \\
\hline P29.3 Persistent fetal circulation & 8 & 0.2 & 86 & 0.1 & $2.43(1.18,5.03)$ \\
\hline Adjusted for Caesarean delivery & & & & & $2.42(1.17,4.99)$ \\
\hline P96 Other conditions originating in the perinatal period & 157 & 4.8 & 1086 & 1.3 & $3.91(3.30,4.65)$ \\
\hline P96.1 Neonatal withdrawal symptoms from maternal use of drugs of addiction & 40 & 1.2 & 206 & 0.2 & $5.12(3.64,7.20)$ \\
\hline P96.2 Withdrawal symptoms from therapeutic use of drugs in newborn & 8 & 0.2 & 8 & 0.0 & $26.18(9.82,69.80)$ \\
\hline P96.8 Other specified conditions originating in the perinatal period & 108 & 3.3 & 837 & 1.0 & $3.45(2.82,4.23)$ \\
\hline
\end{tabular}

was 'P22 respiratory distress of newborn' (OR $1.7 ; 95 \%$ CI $1.5,2.0$ ) for $7.8 \%$ of all children in the SSRI group versus $4.7 \%$ of all other children. This is often seen in infants delivered by Caesarean section; however, after adjusting for the type of delivery there was still an increased risk (OR $1.7 ; 95 \%$ CI 1.5, 2.0). This was not seen in the preterm births. Respiratory and cardiovascular disorders specific to the perinatal period (P20P29) accounted for $13.8 \%$ of the diagnoses reported on the birth admission for term births in the SSRI group (OR 1.8; 95\% CI 1.6, 2.0) versus $8.2 \%$ in the other children. The admissions, 'P29.3 persistent fetal circulation, delayed closure of ductus arteriosus, pulmonary hypertension of newborn (persistent)' comprised $0.2 \%(\mathrm{~N}=8)$ of children in the SSRI group and $0.1 \%(\mathrm{~N}=86)$ of the nonSSRI group term birth admissions (OR 2.4; $95 \%$ CI 1.2, 5.0). After adjusting for Caesarean delivery, the risk of a child in the SSRI group born at term with a diagnosis code of P29.3 being assigned remained at OR 2.4; 95\% CI 1.2, 5.0.

The diagnosis for term births with the greatest risk seen in the children in the SSRI group was 'P96 other conditions originating in the perinatal period' (OR 3.9; 95\% CI 3.3, 4.6). These codes 
were 'P96.1 neonatal withdrawal symptoms from maternal use of drugs of addiction' (OR 5.1; $95 \%$ CI 3.6, 7.2), 'P96.2 withdrawal symptoms from therapeutic use of drugs in newborn' (OR 26.2; 95\% CI 9.8, 69.8) and 'P96.8 other specified conditions originating in the perinatal period' (OR 3.4; 95\% CI 2.8, 4.2).

\section{Non-Birth Admissions Up to 2 Years}

Children born to a mother dispensed an SSRI during her pregnancy were more likely to be admitted to an ICU during the first year of life (OR $1.4 ; 95 \%$ CI $1.3,1.6)$ and also in the second year of life (OR 1.3; 95\% CI 1.1, 1.6) [table III]. There was no difference in the LOS in ICU. Before the age of 1 year, $32.8 \%$ of the children in the SSRI group were admitted to hospital after the birth admission, compared with $24.8 \%$ of the non-SSRI group (OR 1.5; 95\% CI 1.4, 1.6). The most common principal diagnosis was ' $\mathrm{J} 21$ acute bronchiolitis' (OR 1.6; 95\% CI 1.3, 1.8) for $5.9 \%$ of all children in the SSRI group versus $3.8 \%$ of all other children (table V) and this remained a higher risk when analysing just the non-smokers (OR 1.7; 95\% CI 1.4, 2.0). The risk of admission was greater in the SSRI group for eight of the ten most common diagnoses. The admissions were also analysed separately for children of mothers who had smoked or not smoked during the pregnancy, as $28.6 \%$ of the mothers in the SSRI group had smoked during their pregnancy compared with $17.2 \%$ of the other mothers. Within the non-smoking group of mothers, the risk of admission increased for all of the ten most common

Table V. Leading ten principal diagnosis codes for children in the selective serotonin reuptake inhibitor group and born at term, non-birth admissions to 2 years, by number of children admitted, and whether the mother smoked during her pregnancy

\begin{tabular}{|c|c|c|c|c|c|c|c|}
\hline & \multicolumn{2}{|c|}{$\begin{array}{l}\text { SSRIS } \\
\mathrm{N}=2797\end{array}$} & \multicolumn{2}{|c|}{$\begin{array}{l}\text { Non-SSRls } \\
\mathrm{N}=69125\end{array}$} & \multirow{2}{*}{$\begin{array}{l}\text { Admission } \\
\text { OR }(95 \% \mathrm{Cl})\end{array}$} & \multirow{2}{*}{$\begin{array}{l}\text { Mother } \\
\text { non-smoker } \\
\text { during pregnancy } \\
\text { OR }(95 \% \mathrm{Cl})\end{array}$} & \multirow{2}{*}{$\begin{array}{l}\text { Mother } \\
\text { smoked } \\
\text { during pregnancy } \\
\text { OR }(95 \% \mathrm{Cl})\end{array}$} \\
\hline & $\overline{\mathrm{N}}$ & $\%$ & $\overline{\mathrm{N}}$ & $\%$ & & & \\
\hline Children live born 2002-4 & 2797 & & 69125 & & & & \\
\hline Mother smoked during pregnancy & 800 & 28.6 & 11900 & 17.2 & & & \\
\hline $\begin{array}{l}\text { Children with a non-birth admission } \\
\leq 2 \text { years }\end{array}$ & 1200 & 42.9 & 23576 & 34.1 & $1.45(1.34,1.57)$ & $1.44(1.31,1.58)$ & $1.35(1.17,1.56)$ \\
\hline Mother smoked during pregnancy & 369 & 46.1 & 4619 & 38.8 & $1.35(1.17,1.56)$ & & \\
\hline Non-smokers & 831 & 41.6 & 18957 & 33.1 & $1.44(1.31,1.58)$ & & \\
\hline \multicolumn{8}{|l|}{ Principal diagnosis } \\
\hline J21 Acute bronchiolitis & 164 & 5.9 & 2645 & 3.8 & $1.57(1.33,1.84)$ & $1.67(1.37,2.04)$ & $1.11(0.84,1.46)$ \\
\hline $\begin{array}{l}\text { Z76 Persons encountering health } \\
\text { services in other circumstances }\end{array}$ & 150 & 5.4 & 2490 & 3.6 & $1.52(1.28,1.80)$ & $1.59(1.30,1.94)$ & $1.20(0.88,1.64)$ \\
\hline G47 Sleep disorders & 95 & 3.4 & 1527 & 2.2 & $1.56(1.26,1.92)$ & $1.64(1.30,2.07)$ & $1.92(1.14,3.25)$ \\
\hline H65 Nonsuppurative otitis media & 84 & 3.0 & 1276 & 1.8 & $1.65(1.32,2.06)$ & $1.67(1.30,2.16)$ & $1.92(1.18,3.11)$ \\
\hline $\begin{array}{l}\text { J06 Acute upper respiratory } \\
\text { infections of multiple and unspecified } \\
\text { sites }\end{array}$ & 73 & 2.6 & 1332 & 1.9 & $1.36(1.07,1.73)$ & $1.45(1.08,1.93)$ & $1.01(0.66,1.54)$ \\
\hline $\begin{array}{l}\text { R68 Other general symptoms and } \\
\text { signs }\end{array}$ & 64 & 2.3 & 945 & 1.4 & $1.69(1.31,2.18)$ & $1.83(1.39,2.41)$ & $1.87(0.93,3.75)$ \\
\hline $\begin{array}{l}\text { A09 Diarrhoea and gastroenteritis of } \\
\text { presumed infectious origin }\end{array}$ & 61 & 2.2 & 1341 & 1.9 & $1.13(0.87,1.46)$ & $1.24(0.91,1.70)$ & $0.76(0.48,1.22)$ \\
\hline B34 Viral infection of unspecified site & 59 & 2.1 & 1127 & 1.6 & $1.30(1.00,1.69)$ & $1.29(0.94,1.77)$ & $1.22(0.76,1.96)$ \\
\hline $\begin{array}{l}\text { P07 Disorders related to short } \\
\text { gestation and low birth weight, not } \\
\text { elsewhere classified }\end{array}$ & 59 & 2.1 & 851 & 1.2 & $1.73(1.32,2.26)$ & $1.37(0.96,1.97)$ & $2.26(1.50,3.41)$ \\
\hline $\begin{array}{l}\text { A08 Viral and other specified } \\
\text { intestinal infections }\end{array}$ & 48 & 1.7 & 805 & 1.2 & $1.48(1.10,1.99)$ & $1.50(1.06,2.14)$ & $1.30(0.76,2.22)$ \\
\hline
\end{tabular}


diagnoses and was statistically significant for seven out of the ten most common diagnoses in the children of the women in the SSRI group. In the smoking group, the risk of admission remained statistically significantly greater for three of the ten diagnoses.

\section{Additional Diagnoses in Non-Birth Admissions up to 2 Years}

Additional diagnoses were recorded on $53.1 \%$ of the non-birth admission records for the children in the SSRI group compared with $49.7 \%$ of the records for the non-SSRI group (OR 1.2: 95\% CI 1.0, 1.3) [table VI]. The most common diagnosis recorded for both groups of children was 'E86 volume depletion' (OR 1.3; 95\% CI 1.1, 1.7). 'E83 disorders of mineral metabolism' was the diagnosis recorded with the greatest risk of admission in the children in the SSRI group (OR 6.2 ; 95\% CI 2.3, 16.5). 'H65 nonsuppurative otitis media' was also an increased risk for the children in the SSRI group (OR 1.8; 95\% CI 1.3, 2.4).

\section{Discussion}

Antidepressants are dispensed to a large number of pregnant women and we have previously published data on the dispensing patterns and the characteristics of the WA women dispensed an
SSRI during their pregnancy. ${ }^{[13]}$ It is important to have follow-up information on the developmental well-being of the children exposed to SSRIs in utero. This is the first population-based study in Australia to examine the early hospital admissions of the children born to women dispensed an SSRI during their pregnancy. In this cohort, an SSRI was dispensed to 3703 (3.8\%) pregnant women with 3764 children in utero. It is also one of the largest studies of pregnant women using SSRIs to follow the children through to 2 years of age to investigate morbidity and mortality.

Our study shows that children born to women dispensed an SSRI during their pregnancy were still more likely to be born preterm than children born to women not dispensed an SSRI during pregnancy after adjusting for any previous preterm births, whether the mother smoked during her pregnancy, SEIFA, parity and maternal age. Their mean birth weight was $100 \mathrm{~g}$ less than the mean weight of children in the non-SSRI group. Following the birth admission and before the age of 2 years, the children in the SSRI group were more likely to be admitted for bronchiolitis, which remained a significant risk for admission after stratifying by whether the mother smoked during her pregnancy. The mothers who smoked during their pregnancy probably continued to smoke around their child in the home. However,

Table VI. Leading ten additional diagnosis codes for non-birth admissions to 2 years, by number of children admitted

\begin{tabular}{|c|c|c|c|c|c|}
\hline & \multicolumn{2}{|c|}{$\begin{array}{l}\text { SSRIS } \\
\mathrm{N}=2797\end{array}$} & \multicolumn{2}{|c|}{$\begin{array}{l}\text { Non-SSRIs } \\
\mathrm{N}=69125\end{array}$} & \multirow{2}{*}{$\begin{array}{l}\text { Admission } \\
\text { OR }(95 \% \mathrm{Cl})\end{array}$} \\
\hline & $\mathrm{N}$ & $\%$ & $\mathrm{~N}$ & $\%$ & \\
\hline Children with a non-birth admission $\leq 2$ years & 1200 & 42.9 & 23576 & 34.1 & $1.45(1.34,1.57)$ \\
\hline Children with an additional diagnosis recorded & 637 & 53.1 & 11711 & 49.7 & $1.15(1.02,1.29)$ \\
\hline E86 Volume depletion & 83 & 3.0 & 1555 & 2.2 & $1.33(1.06,1.66)$ \\
\hline B96 Other bacterial agents as the cause of diseases classified to other chapters & 59 & 2.1 & 1000 & 1.4 & $1.47(1.13,1.91)$ \\
\hline J06 Acute upper respiratory infections of multiple and unspecified sites & 46 & 1.6 & 787 & 1.1 & $1.45(1.08,1.96)$ \\
\hline P07 Disorders related to short gestation and low birth weight, not elsewhere classified & 46 & 1.6 & 703 & 1.0 & $1.63(1.20,2.20)$ \\
\hline R63 Symptoms and signs concerning food and fluid intake & 43 & 1.5 & 803 & 1.2 & $1.33(0.98,1.81)$ \\
\hline H66 Suppurative and unspecified otitis media & 43 & 1.5 & 596 & 0.9 & $1.80(1.31,2.45)$ \\
\hline K21 Gastro-oesophageal reflux disease & 41 & 1.5 & 642 & 0.9 & $1.59(1.15,2.18)$ \\
\hline R68 Other general symptoms and signs & 40 & 1.4 & 680 & 1.0 & $1.46(1.06,2.01)$ \\
\hline P92 Feeding problems of newborn & 39 & 1.4 & 612 & 0.9 & $1.58(1.14,2.19)$ \\
\hline $\begin{array}{l}\text { B95 Streptococcus and Staphylococcus as the cause of diseases classified to other } \\
\text { chapters }\end{array}$ & 38 & 1.4 & 632 & 0.9 & $1.49(1.07,2.08)$ \\
\hline
\end{tabular}

$\mathbf{O R}=$ odds ratio; $\mathbf{S S R}=$ selective serotonin reuptake inhibitor. 
the mothers who didn't smoke during their pregnancy had a higher risk of their child being admitted for bronchiolitis than the mothers who did smoke (OR 1.7; 95\% CI 1.4, 2.0 vs OR 1.1; 95\% CI 0.8, 1.5) when compared with the nonSSRI mothers. The next more likely admission was for "persons encountering health services in other circumstances' - a category usually assigned when a healthy child is admitted due to family circumstances. These admissions may be related to the underlying depressive condition of the mother and her ability to cope with a newborn.

The main limitation of using dispensing data relates to whether the medicine was consumed, or consumed as directed, and we have no information in this study for either of these aspects of use. It is not known whether the mother actually ingested the medicine but by reviewing the repeat dispensing of SSRIs throughout the trimesters of pregnancy, adherence to the medicines may be inferred. $75.4 \%$ of the women were dispensed an SSRI in at least two consecutive trimesters, from $\mathrm{T} 0$ to $\mathrm{T} 3,{ }^{[13]}$ and this would indicate the women are using the SSRIs as each dispensing costs from \$A3.60 to \$A4.60 per medicine for concessional patients and from \$A22.40 to \$A28.60 for general patients, during the period of the study. The mean number of dispenses was 2.2 in $\mathrm{T} 1$ and 4.4 overall. If the depression per se is the reason for the increased risks and women with depression are not treated with SSRIs or treated with nonSSRI medicines, the effects observed in our study would be understated as those women would be in the non-SSRI group. Whilst the study is based upon births in WA from 2002 to 2005, the SSRI medications analysed are still the most commonly prescribed SSRIs given to pregnant women. Although the use of administrative data may not provide a complete picture of the factors associated with the use of medications in pregnancy, the approach provides a rich source of information on pregnancy outcomes in large populations.

A review of perinatal depression concluded that, although limited, the available research suggests that depression is one of the most common perinatal complications. ${ }^{[27]}$ Since 2006, the American College of Obstetricians and Gynecologists has recommended screening for depression during each trimester of pregnancy. ${ }^{[28]}$ The first Australian Clinical Practice Guidelines surrounding the detection, management and treatment of perinatal mental health disorders were established in 2011. ${ }^{[29]}$ Australian studies have also highlighted the need for screening pregnant women. ${ }^{[30,31]}$ The risks of allowing a depressive episode to remain untreated may be far greater than those associated with antidepressant treatment. ${ }^{[3]}$ Depression may increase the risk of self-injurious or suicidal behaviours in the mother but also may contribute to inadequate self-care and poor compliance with prenatal care. ${ }^{[32]}$ Women with depression often present with decreased appetite and consequently lower-than-expected weight gain in pregnancy, factors that have been associated with negative pregnancy outcomes. ${ }^{[32,33]}$ In addition, persons with a major depressive disorder are twice as likely to smoke as other persons ${ }^{[34-36]}$ and to use either alcohol or illicit drugs, ${ }^{[33]}$ behaviours that further increase risk to the foetus. ${ }^{[37]}$ Although it has been difficult to assess the impact of antenatal depression on foetal development and neonatal well-being in humans, several studies have found an association between maternal depression and factors that predict poor neonatal outcome, including preterm birth, lower birth weight, smaller head circumference and lower APGAR scores. ${ }^{[37-43]}$ A recent study by Ponder et al. ${ }^{[4]}$ found that maternal depression/anxiety affected a particular gene expression in the placenta suggesting a possible mechanism for the effects of maternal mood on foetal neurodevelopmental programming. Studies have shown that SSRIs readily cross the placenta, ${ }^{[6]}$ are present in amniotic fluid ${ }^{[7]}$ and newborns exposed to them in utero may experience withdrawal-like symptoms after birth. ${ }^{[8,9]}$

The FDA have issued a Drug Safety Communication (14 December 2011) related to the use of SSRIs during pregnancy and the potential risk of a rare heart and lung condition known as persistent pulmonary hypertension of the newborn (PPHN) ${ }^{[45]}$ The initial Public Health Advisory in July 2006 on this potential risk was based on a single published study. ${ }^{[46]}$ Since then, there have been conflicting findings from new studies evaluating this potential risk, making it unclear 
whether use of SSRIs during pregnancy can cause PPHN. Two studies suggest an increased risk for PPHN with SSRI use in pregnancy ${ }^{[46,47]}$ and three studies reported no increase in risk of PPHN. ${ }^{[48-50]}$ The FDA has reviewed the additional new study results and has concluded that, given the conflicting results from different studies, it is premature to reach any conclusion about a possible link between SSRI use in pregnancy and PPHN. It was noted by the FDA communication that PPHN typically presents in term or late-preterm births, and that Caesarean delivery was a potentially important factor. These admissions would most likely have been coded in our data as 'P29.3 persistent fetal circulation, delayed closure of ductus arteriosus, pulmonary hypertension of newborn (persistent)'. In term births, our study had 8 children in the SSRI group and 86 in the non-SSRI group with this as the principal diagnosis or as an additional diagnosis recorded on their birth admission (OR 2.4; 95\% CI 1.2, 5.0). After adjusting for Caesarean delivery, the risk did not change. A very large population-based study in five Nordic countries of births from 1996 to 2007 has been published since the FDA communication. They reported 33 children in the SSRI group with PPHN with an unadjusted OR of $2.5 ; 95 \%$ CI 1.8, 3.6. ${ }^{[51]}$ The absolute risk of PPHN is still low, and the P29.3 code also includes patent ductus arteriosus.

\section{Conclusion}

Separating the effects of SSRI treatment from the effects of the depression alone in the mothers is an area for further research. The main limitation of this study is that we can only use the dispensing of an SSRI as a marker for depression and anxiety in the mother.

Assessing the impact of antenatal depression on the foetus, as well as the mother, requires a balance of risks and benefits in the use of SSRIs. Clinicians may need to counsel their patients regarding drinking habits, smoking, weight gain and other factors that are known to increase the risks of poor neonatal outcomes, especially those factors that increase the risk of preterm birth.
We found 'P20-P29 respiratory and cardiovascular disorders specific to the perinatal period' accounted for $13.8 \%$ of the diagnoses reported on the birth admission for term births in the SSRI group (OR 1.8; 95\% CI 1.6, 2.0) with more than half of these coded as ' $\mathrm{P} 22$ respiratory distress of newborn'. We did not find a similar increase in the preterm births.After adjusting for Caesarean delivery, in infants exposed to SSRIs in pregnancy compared with non-exposed infants, there was an increased risk of being assigned a diagnosis code of P29.3 (PPHN) [OR 2.4; 95\% CI 1.2, 5.0].

The increased risk of death in the first year of life in the children in the SSRI group is a new finding (OR 1.8; 95\% CI 1.3, 2.6), although it is based upon very small numbers. Of the SSRIs used as monotherapy, paroxetine had the highest risk of death in live birth infants (OR 2.2; $95 \%$ CI $1.0,4.6)$ The categorization code for death 'P01 fetus and newborn affected by maternal complications of pregnancy' did not reach statistical significance (OR 2.1; 95\% CI 0.5, 8.9) but it is interesting that this is twice as likely to be the most common code used for death in the SSRI group compared with the non-SSRI group. Nearly every finding of a risk increase, if not extremely strong, should only be regarded as a tentative signal to be tested in independent studies. ${ }^{[52]}$ Further work is needed to investigate whether these increased risks in mortality and morbidity are due to prenatal exposure to SSRIs, to the maternal depression itself, to SSRI use being a proxy for an environmental exposure such as smoking or to a combination of these factors. The dilemma for researchers is in designing a study that can account for these and other confounders, as a randomized clinical trial is not possible. In the meantime, the clinician and the woman herself need to balance the degree of severity of the depressive disorder and the risk of relapse, with the emerging safety profile of the individual SSRI.

\section{Acknowledgements}

The authors report no conflicts of interest in this work. This project has approval from the Human Research Ethics Committee of The University of Western Australia (ref \#RA/4/1/1390) and was performed under the auspices of the WA Cross Jurisdictional Data Linkage Project covered by a 
Memorandum of Understanding between the Western Australia Department of Health and the Australian Government Department of Health and Ageing. The person-based linkage was covered by approvals from the two agencies' ethics and confidentiality committees. Before the event-based data linkage was undertaken, approval was also obtained from the Confidentiality of Health Information Committee (approval \#200534) and permission to use the required data was obtained from the relevant data custodians. Carol Bower was funded by a National Health and Medical Research Fellowship (\#634341).

\section{References}

1. American College of Obstetricians and Gynecologists. ACOG practice bulletin no. 92: use of psychiatric medications during pregnancy and lactation. Obstet Gynecol 2008; 111 (4): 1001-20

2. Buist A, Ellwood D, Brooks J, et al. National program for depression associated with childbirth: the Australian experience. Best Pract Res Clin Obstet Gynaecol 2007 Apr; 21 (2): 193-206

3. Cohen LS, Altshuler LL, Harlow BL, et al. Relapse of major depression during pregnancy in women who maintain or discontinue antidepressant treatment. JAMA 2006 Feb; 295 (5): 499-507

4. Field T, Diego M, Hernandez-Reif M, et al. Sleep disturbances in depressed pregnant women and their newborns. Infant Behav Dev 2007; 30 (1): 127-33

5. Field T, Diego M, Hernandez-Reif M. Prenatal depression effects and interventions: a review. Infant Behav Dev 2010 Dec; 33 (4): 409-18

6. Hendrick V, Stowe ZN, Altshuler LL, et al. Placental passage of antidepressant medications. Am J Psychiatry 2003 May; 160 (5): 993-6

7. Loughhead AM, Fisher AD, Newport DJ, et al. Antidepressants in amniotic fluid: another route of fetal exposure. Am J Psychiatry 2006 Jan; 163 (1): 145-7

8. Koren G, Matsui D, Einarson A, et al. Is maternal use of selective serotonin reuptake inhibitors in the third trimester of pregnancy harmful to neonates? CMAJ 2005 May; 172 (11): $1457-9$

9. Moses-Kolko EL, Bogen D, Perel J, et al. Neonatal signs after late in utero exposure to serotonin reuptake inhibitors: literature review and implications for clinical applications. JAMA 2005 May; 293 (19): 2372-83

10. Belik J. Fetal and neonatal effects of maternal drug treatment for depression. Semin Perinatol 2008; 32 (5): 350-4

11. Gentile S, Galbally M. Prenatal exposure to antidepressant medications and neurodevelopmental outcomes: a systematic review. J Affect Disord 2011 Mar 18; 128 (1-2): 1-9

12. Gentile S. On categorizing gestational, birth, and neonatal complications following late pregnancy exposure to antidepressants: the prenatal antidepressant exposure syndrome. CNS Spectr 2010 Mar; 15 (3): 167-85

13. Colvin L, Slack-Smith L, Stanley FJ, et al. Dispensing patterns and pregnancy outcomes for women dispensed selective serotonin reuptake inhibitors in pregnancy. Birt Defects Res A Clin Mol Teratol 2011; 91 (3): 142-52

14. Colvin L, Slack-Smith L, Stanley FJ, et al. Pharmacovigilance in pregnancy using population-based linked da- tasets. Pharmacoepidemiol Drug Saf 2009 Jan; 18 (3): 211-25

15. Colvin L, Slack-Smith L, Stanley FJ, et al. Linking a pharmaceutical claims database with a birth defects registry to investigate birth defect rates of suspected teratogens. Pharmacoepidemiol Drug Saf 2010 Jul; 19 (11): 1137-50

16. Australian Government Department of Health and Ageing. Pharmaceutical benefits scheme (PBS) [online]. Available from URL: http://www.pbs.gov.au/ [Accessed 2010 Oct 2]

17. National Centre for Classification in Health. International statistical classification of diseases and related health problems, tenth revision, Australian modification (ICD10-AM). Sydney (NSW): National Centre for Classification in Health, 1999

18. Department of Health Western Australia. Hospital morbidity data system reference manual, July 2004. Perth (WA): Health Data Collections Branch, Health Information Centre, 2004

19. Australian Bureau of Statistics. Socio-economic indexes for areas (SEIFA): technical paper 2006. Report no. 2039.0.55.001. Canberra (ACT): Commonwealth of Australia, 2008

20. Blair E, Liu Y, Cosgrove P. Choosing the best estimate of gestational age from routinely collected population-based perinatal data. Paediatr Perinat Epidemiol 2004 Jul; 18 (4): 270-6

21. Milne E, Laurvick CL, Blair E, et al. Fetal growth and acute childhood leukemia: looking beyond birth weight. Am J Epidemiol 2007 Jul; 166 (2): 151-9

22. United Nations, Department of Economic and Social Affairs, Population Division. World population prospects: the 2010 revision, CD-ROM edition. File 6-1: infant mortality rate (both sexes combined) by major area, region and country, 1950-2100 (infant deaths per 1,000 live births). New York (NY): United Nations, 2011

23. Brant R. Inference for a single proportion: comparing to a known proportion [online]. Available from URL: http:// www.stat.ubc.ca/ rollin/stats/ssize/b1.html [Accessed 2012 Mar 23]

24. Holman CD, Bass AJ, Rouse IL, et al. Population-based linkage of health records in Western Australia: development of a health services research linked database. Aust N Z J Public Health 1999 Oct; 23 (5): 453-9

25. Bower C, Silva D, Henderson TR, et al. Ascertainment of birth defects: the effect on completeness of adding a new source of data. J Paediatr Child Health 2000 Dec; 36 (6): 574-6

26. Giles GG. Medical record linkage in Australia: this is as good as it gets. ANZ J Surg 2005 May; 75 (5): 259

27. Gaynes BN, Gavin N, Meltzer-Brody S, et al. Perinatal depression: prevalence, screening accuracy, and screening outcomes. Evid Rep Technol Assess (Full Rep) 2005; (119): $1-225$

28. American College of Obstetricians and Gynecologists. ACOG committee opinion no. 343: psychosocial risk factors: perinatal screening and intervention. Obstet Gynecol 2006 Aug; 108 (2): 469-77

29. Austin MP, Highet N, the Guidelines Expert Advisory Committee. Clinical practice guidelines for depression and related disorders, anxiety, bipolar disorder and puerperal 
psychosis, in the perinatal period: a guideline for primary care health professionals. Melbourne (VIC): beyondblue: The National Depression Initiative, 2011

30. Buist AE, Austin MP, Hayes BA, et al. Postnatal mental health of women giving birth in Australia 2002-2004: findings from the beyondblue National Postnatal Depression Program. Aust N Z J Psychiatry 2008 Jan; 42 (1): 66-73

31. Jones CJ, Creedy DK, Gamble JA. Australian midwives' awareness and management of antenatal and postpartum depression. Women Birth 2011; 25 (1): 23-8

32. Woods SM, Melville JL, Guo YQ, et al. Psychosocial stress during pregnancy. Am J Obstet Gynecol 2010 Jan; 202 (1): 61.e1-7

33. Zuckerman B, Amaro H, Bauchner H, et al. Depressive symptoms during pregnancy: relationship to poor health behaviors. Am J Obstet Gynecol 1989 May; 160 (5 Pt 1): 1107-11

34. Breslau N. Psychiatric comorbidity of smoking and nicotine dependence. Behav Genet 1995 Mar; 25 (2): 95-101

35. Lasser K, Boyd JW, Woolhandler S, et al. Smoking and mental illness: a population-based prevalence study. JAMA 2000 Nov; 284 (20): 2606-10

36. Grant BF, Hasin DS, Chou SP, et al. Nicotine dependence and psychiatric disorders in the United States: results from the National Epidemiologic Survey on Alcohol and Related Conditions. Arch Gen Psychiatry 2004 Nov; 61 (11): 1107-15

37. Nonacs R, Cohen LS. Assessment and treatment of depression during pregnancy: an update. Psychiatr Clin North Am 2003 Sep; 26 (3): 547-62

38. Orr ST, Miller CA. Maternal depressive symptoms and the risk of poor pregnancy outcome: review of the literature and preliminary findings. Epidemiol Rev 1995; 17 (1): $165-71$

39. Orr ST, James SA, Blackmore Prince C. Maternal prenatal depressive symptoms and spontaneous preterm births among African-American women in Baltimore, Maryland. Am J Epidemiol 2002 Nov; 156 (9): 797-802

40. Dayan J, Creveuil C, Herlicoviez M, et al. Role of anxiety and depression in the onset of spontaneous preterm labor. Am J Epidemiol 2002 Feb; 155 (4): 293-301

41. Steer RA, Scholl TO, Hediger ML, et al. Self-reported depression and negative pregnancy outcomes. J Clin Epidemiol 1992 Oct; 45 (10): 1093-9

42. Zuckerman B, Bauchner H, Parker S, et al. Maternal depressive symptoms during pregnancy, and newborn irritability. J Dev Behav Pediatr 1990 Aug; 11 (4): 190-4
43. Alder J. Depression and anxiety during pregnancy: a risk factor for obstetric, fetal and neonatal outcome? A critical review of the literature. J Matern Fetal Neonatal Med 2007 Mar; 20 (3): 189-209

44. Ponder KL, Salisbury A, McGonnigal B, et al. Maternal depression and anxiety are associated with altered gene expression in the human placenta without modification by antidepressant use: implications for fetal programming. Dev Psychobiol 2011; 53 (7): 711-23

45. US Food and Drug Administration. FDA drug safety communication: selective serotonin reuptake inhibitor (SSRI) antidepressant use during pregnancy and reports of a rare heart and lung condition in newborn babies [online]. Available from URL: http://www.fda.gov/drugs/drugsaf ety/ucm283375.htm [Accessed 2011 Dec 21]

46. Chambers CD, Hernandez-Diaz S, Van Marter LJ, et al. Selective serotonin-reuptake inhibitors and risk of persistent pulmonary hypertension of the newborn. N Engl J Med 2006 Feb; 354 (6): 579-87

47. Källén B, Olausson PO. Maternal use of selective serotonin re-uptake inhibitors and persistent pulmonary hypertension of the newborn. Pharmacoepidemiol Drug Saf 2008 Aug; 17 (8): 801-6

48. Wichman CL, Moore KM, Lang TR, et al. Congenital heart disease associated with selective serotonin reuptake inhibitor use during pregnancy. Mayo Clin Proc 2009; 84 (1): 23-7

49. Andrade SE, McPhillips H, Loren D, et al. Antidepressant medication use and risk of persistent pulmonary hypertension of the newborn. Pharmacoepidemiol Drug Saf 2009; 18 (3): 246-52

50. Wilson KL, Zelig CM, Harvey JP, et al. Persistent pulmonary hypertension of the newborn is associated with mode of delivery and not with maternal use of selective serotonin reuptake inhibitors. Am J Perinatol 2011 Jan; 28 (1): 19-24

51. Kieler H, Artama M, Engeland A, et al. Selective serotonin reuptake inhibitors during pregnancy and risk of persistent pulmonary hypertension in the newborn: population based cohort study from the five Nordic countries. BMJ 2012; 344: d8012

52. Källén BA. Methodological issues in the epidemiological study of the teratogenicity of drugs. Congenit Anom (Kyoto) 2005 Jun; 45 (2): 44-51

Correspondence: Lyn Colvin, Telethon Institute for Child Health Research, PO Box 855, West Perth, WA 6872, Australia. E-mail: lync@ichr.uwa.edu.au 